\title{
BMJ Open Assessment of the effects of methodological choice in continuity of care research: a real-world example with dyslipidaemia cohort
}

\author{
Eunjung Choo, ${ }^{1}$ Eunyoung Choi, ${ }^{2,3}$ Juhee Lee, ${ }^{4}$ Linda Siachalinga ${ }^{2}$ Eun Jin Jang, ${ }^{5}$ \\ lyn-Hyang Lee (D) ${ }^{2}$
}

To cite: Choo E, Choi E, Lee J, et al. Assessment of the effects of methodological choice in continuity of care research: a real-world example with dyslipidaemia cohort. BMJ Open 2021;11:e053140. doi:10.1136/ bmjopen-2021-053140

- Prepublication history and additional supplemental material for this paper are available online. To view these files, please visit the journal online (http://dx.doi.org/10.1136/ bmjopen-2021-053140).

EChoo and EChoi contributed equally.

Received 05 May 2021

Accepted 08 December 2021

Check for updates

(c) Author(s) (or their employer(s)) 2021. Re-use permitted under CC BY-NC. No commercial re-use. See rights and permissions. Published by BMJ.

${ }^{1}$ College of Pharmacy, Ajou University, Suwon, Korea

${ }^{2}$ College of Pharmacy, Yeungnam University, Gyeongsan, Korea

${ }^{3}$ Department of Pharmacy, Ulsan University Hospital, Ulsan, Korea

${ }^{4}$ Department of Statistics,

Kyungpook National University, Daegu, Korea

${ }^{5}$ Department of Information Statistics, Andong National University, Andong, Korea

Correspondence to Dr lyn-Hyang Lee; leeiynhyang@ynu.ac.kr and Dr Eun Jin Jang;

ejjang@anu.ac.kr

\section{ABSTRACT}

Objective To determine if the choice of methodological elements affects the results in continuity of care studies. Design This is a retrospective cohort study. The association between continuity of care and clinical outcome was investigated using the Continuity of Care Index. The association was explored in 12 scenarios based on four definitions of the relative timing of continuity and outcome measurements in three populations (three PS $\times$ four $T \mathrm{~s}$ ).

Setting National Health Insurance claims from all primary and secondary care facilities in South Korea between 2007 and 2015.

Participants Participants were patients diagnosed with dyslipidaemia, made $\geq 2$ ambulatory visits and were newly prescribed with $\geq 1$ antihyperlipidaemic agent at an ambulatory setting in 2008. Three study populations were defined based on the number of ambulatory visits: 10084 patients in population 1 (P1), 8454 in population 2 (P2) and 4754 in population $3(\mathrm{P} 3)$.

Main outcome measure Hospitalisation related to one of the four atherosclerotic cardiovascular diseases, including myocardial infarction, stable or unstable angina, ischaemic stroke and transient ischaemic attack.

Results Concurrent measure of continuity and outcome (T1) showed a significantly higher risk of hospitalisation (adjusted HRs: 2.73-3.07, p<0.0001) in the low continuity of care group, whereas $\mathrm{T} 2$, which measured continuity until the outcome occurred, showed no risk difference between the continuity of care groups. T3, which measured continuity as a time-varying variable, had adjusted HRs of $1.31-1.55(p<0.05)$, and T4, measuring continuity for a predefined period and measuring outcomes in the remaining period, had adjusted HRs of $1.34-1.46(p<0.05)$ in the low continuity of care. Within each temporal relationship, the effect estimates became more substantial as the inclusion criteria became stricter.

Conclusions The study design in continuity of care studies should be planned carefully because the results are sensitive to the temporal relationship between continuity and outcome and the population selection criteria.
Strengths and limitations of this study

- This is the first study to employ empirical data to demonstrate the magnitude of potential bias that could arise due to inadequate definitions of the relative timing between continuity and outcome measures.

- This study used representative sample data (sample size $=1035$ 089) from national claims data covering the entire Korean population.

- Twelve different analysis scenarios were used to estimate the association between continuity of care and clinical outcomes.

- This study could not include data on potentially relevant covariates, such as those for the quality of the relationship and the level of informational link between different providers, due to the administrative nature of claims data

- Korean-specific medical culture and the administrative nature of claims data limit the generalisability of the study.

\section{INTRODUCTION}

Many researchers have attempted to quantify the association between continuity of care (COC) and patient outcomes across various health conditions. ${ }^{1}{ }^{2}$ Despite these efforts, some questions on how to define and measure the crucial elements in COC studies remain unanswered. ${ }^{3}{ }^{4}$ In a systematic review published in 2010, van Walraven and colleagues ${ }^{5}$ analysed 18 studies and reported a potential benefit of increasing care continuity in patient experiences and clinical results. On the other hand, they expressed considerable concerns over methodological limitations observed in previous studies during the initial screening. ${ }^{5}$ Of 139 studies, they excluded $59 \%$ of the studies because they measured continuity and outcomes concurrently without an appropriate analysis. van Walraven et a $\tilde{l}$ warned that improper timing of obtaining continuity 
and outcome measures might generate biased results. Notably, even after the review of van Walraven et al, ${ }^{5}$ studies that measured continuity and outcomes concurrently without an appropriate statistical strategy were still published. ${ }^{6-10}$

The most recent systematic review of COC studies showed that the 15 included studies used various inclusion rules on the minimum number of visits, from two to four visits. ${ }^{2}$ This variation may be due to an inherent feature of continuity measurements, for example, the Bice-Boxerman Continuity of Care Index (COCI) or the Sequential Continuity Index (SECON), which can calculate continuity in patients with at least two visits. ${ }^{71} \mathrm{In}$ practice, however, the number of visits needed to develop a valid patient-doctor relationship is debatable. For a patient who visits the same doctor twice in 5 or 10 years, the COCI is 1 regardless of duration; however, it is unclear if the care continuity between the patient and the doctor was established under such conditions. As seen in this simple illustration, if the total number of visits is too low, the situation cannot be considered as actual continuity, even though the quantitatively measured continuity index is high, leading to a biased conclusion.

The present study aimed to empirically explore whether a researcher's choice of definition for the number of visits as an inclusion criterion or the relative timing of continuity and outcome measures can affect the results of continuity analysis. To this end, this study examined the association of COC with four atherosclerotic cardiovascular diseases (ASCVDs)-related hospitalisation in patients with dyslipidaemia in South Korea using healthcare claims data. Three options for the inclusion criteria and four options for the temporal relationship between continuity and outcome measures were established, and the risk of ASCVD-related hospitalisation across the study scenarios was investigated.

\section{METHODS}

\section{Data sources}

The National Sample Cohort (NHIS-NSC) data were analysed. The NHIS-NSC data are released by the Korean National Health Insurance Service (KNHIS) for research purposes and include a $2 \%$ stratified random sample ( $\mathrm{n}=1035$ 089) of all insured patients from 2002 to 2015. The KNHIS provides universal coverage for the Korean population. ${ }^{12}$ The NHIS-NSC data contain anonymised reimbursement data, including individual patient demographic information and information on the utilisation of medical facilities, such as diagnosed health conditions, details of diagnostic tests, prescribed medications and costs. The NHIS-NSC data include diagnoses based on the International Classification of Diseases, 10th version (ICD-10) codes. In addition, it provides KNHIS certificate data, including death records, addresses and level of National Health Insurance (NHI) contributions, which is a proxy for household income.
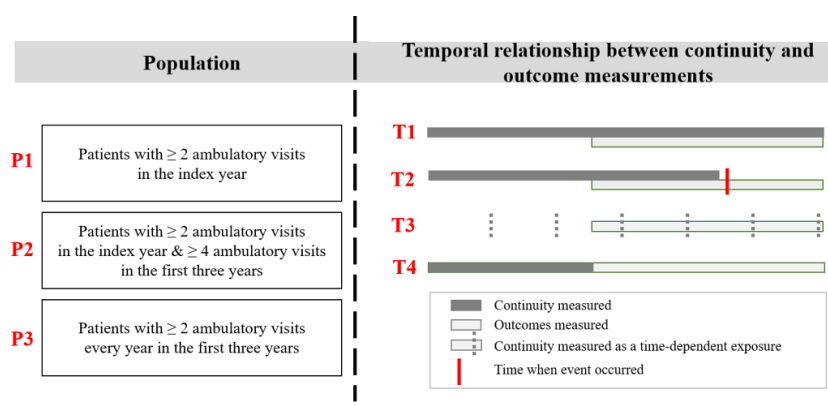
outcome measurements

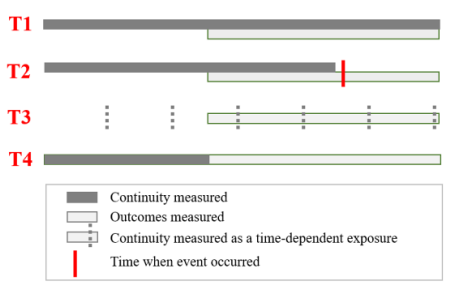

a) Illustration of scenarios explored
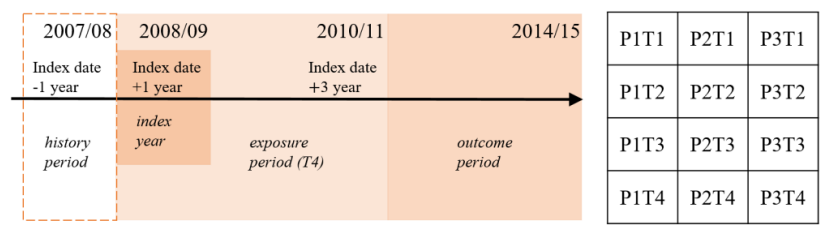

b) Schematic diagram of the time frame for population selection c) 12 study scenarios

Figure 1 Illustration of the study design.

\section{Study design and population}

This was a retrospective cohort study. The degree of association between care continuity and patient clinical outcomes was compared by examining 12 scenarios (figure 1) from a combination of two elements: (1) the number of visits as a criterion excluding extreme low users and (2) the temporal relationship between continuity and outcome measurements.

Patients diagnosed with dyslipidaemia (E78, ICD10), made at least two ambulatory visits and were newly prescribed with at least one antihyperlipidaemic agent at an ambulatory setting during the index year, 2008, were identified. Ambulatory visits made in clinics and outpatient departments of hospitals were included. The index date was defined as the first visit date diagnosed with dyslipidaemia in 2008 . The observational period was 7 years from the index date (figure 1). For example, in the case of a patient identified on 1 January 2008, the observation period would be from 1 January 2008 to 31 December 2014, whereas another patient identified on 1 May 2008 would be observed until 30 April 2015.

Patients who were diagnosed with ischaemic heart diseases (I20-I25), cerebrovascular diseases and related syndromes (I60-I69, G45-G46), diabetes mellitus with circulatory complications (E10.5, E11.5, E12.5, E13.5, E14.5) and cancer (C00-C97) during the history period, 1 year prior to the index date, were excluded. Those who were hospitalised due to one of the four ASCVDs and those who died during the first 3 years after the index date were also excluded. The four ASCVDs were myocardial infarction (I21.0-4, I21.9, I22.0-1, I22.8-9), stable or unstable angina (I20), ischaemic stroke (I63.0-6, I63.8-9) and transient ischaemic attack (G45.0-3, G45.8-9).

Defining three populations based on the number of visits

Three patient inclusion criteria were formed (figure 1): (1) P1: patients who made at least two visits to a doctor during the first 1 year; (2) P2: patients who made at least 
two visits to a doctor during the first 1 year and at least four visits during the first 3 years; and (3) P3: patients who made at least two visits to a doctor every year during the first 3 years.

\section{Temporal relationship between continuity and outcome measurements}

As shown in figure 1, the period measuring continuity is different by scenario option for each patient: (1) T1: continuity was measured during the whole study period; (2) T2: continuity was determined from the data available up to the end of the previous quarter when the outcome event occurred; (3) T3: continuity was measured quarterly and considered as a time-varying variable; and (4) T4: continuity was determined from data available in the first 3 years (ie, exposure period). Technically, for the T2 and T3 options the available data were restructured quarterly from the index date. Continuity was measured during the whole study period for patients having no outcome event within the study period. To measure the outcomes, in all scenarios, each patient was followed up after the 3-year baseline period until death, event or end of study period, whichever occurred first.

\section{Measures}

Continuity of care

Among the known measures of COC, this study used the COCI. ${ }^{11} 1314$ While the Usual Provider Continuity (UPC) or SECON indices can be good options to measure continuity in chronic conditions, the COCI was considered the best choice for the study. The COCI was recommended for use in contexts such as South Korea, where patients may contact many different healthcare providers. ${ }^{14}$ Other options such as the UPC index require identification of a usual care provider, which was not possible with the claims data we analysed. ${ }^{14}$ SECON is useful in assessing the need to share information among providers and is related more to short-term continuity than long-term continuity. ${ }^{7}$

For each patient, the COCI value ranges from 0 to 1 , with an index closer to 1 indicating better continuity. If a patient's COCI is 1 , the patient always consults the same provider. The formula for $\mathrm{COCI}$ is:

$$
\mathrm{COCI}=\frac{\sum_{\mathrm{j}=1}^{\mathrm{s}} \mathrm{n}_{\mathrm{j}}^{2}-\mathrm{N}}{\mathrm{N}(\mathrm{N}-1)}
$$

where $\mathrm{N}$ is the total number of visits; $\mathrm{n}_{\mathrm{j}}$ is the number of visits to provider $\mathrm{j}$; and $\mathrm{s}$ is the number of different providers the patient has visited. ${ }^{11}$ The COCI can only be defined for patients with more than two visits (for $\mathrm{N}=1$ the COCI is not defined because of division by 0 ), which is one of the inclusion criteria of this study.

For each patient, the COCI was calculated based on the distribution of visits among distinct providers. In a primary care setting, a visit to the same doctor's office was considered a visit to the same doctor. In a secondary care setting, a visit to the same medical department in the same hospital was considered a visit to the same doctor.
Continuity cohort

The COCI is sensitive to the number of different doctors a patient visiting. ${ }^{13} 14$ For example, if a patient had ten doctor visits, including nine visits with doctor $\mathrm{A}$, the COCI would be 0.8. Alternatively, if a patient had eight visits with doctor $\mathrm{A}$ and two with doctor $\mathrm{B}$, the COCI would be 0.64 . Similarly, if a patient had eight visits with doctor A and one each with doctors B and C, the COCI would be 0.62 . To the best of our knowledge, there are no commonly used criteria for high and low COC levels. Thus, based on an interim analysis of the COCI distribution within the study population, COCI $\geq 0.8$ and COCI $<0.8$ were defined as indicating high COC and low COC, respectively. From a practical perspective, a patient who made 9 of 10 visits to the same doctor was considered to have a high continuity relationship with that doctor. Our interim analysis showed that the COCI for $63 \%-66 \%$ of the included patients in this study was higher than 0.8 (figure 2).

As an alternative approach, the median value was used as a cut-point to define high and low COC groups and applied to the three populations using the temporal relationship T4. The median COCI was 1.0; patients with a COCI of 1 were classified in the high COC group and patients with a COCI of $<1$ were classified in the low COC group. The COCI cut-off point was determined from the data available during the first 3 years.

\section{Outcomes}

The primary outcome was defined as hospitalisation due to one of the four ASCVDs (myocardial infarction, stable or unstable angina, ischaemic stroke and transient ischaemic attack). Using the diagnosis codes and the relevant diagnostic tests or treatments, the corresponding hospital admissions were identified from the data as follows: (1) myocardial infarction, diagnosed with codes I21.0-4, I21.9, I22.0-1 and I22.8-9 and treated using Percutaneous Coronary Intervention (PCI) or Coronary Artery Bypass Surgery (CABG) or fibrinolytics; (2) stable or unstable angina, diagnosed with codes I20 and treated using PCI or CABG or fibrinolytics; (3) ischaemic stroke, diagnosed with codes I63.0-6 and I63.8-9 and assessed by brain imaging tests (CT or MRI); and (4) transient ischaemic

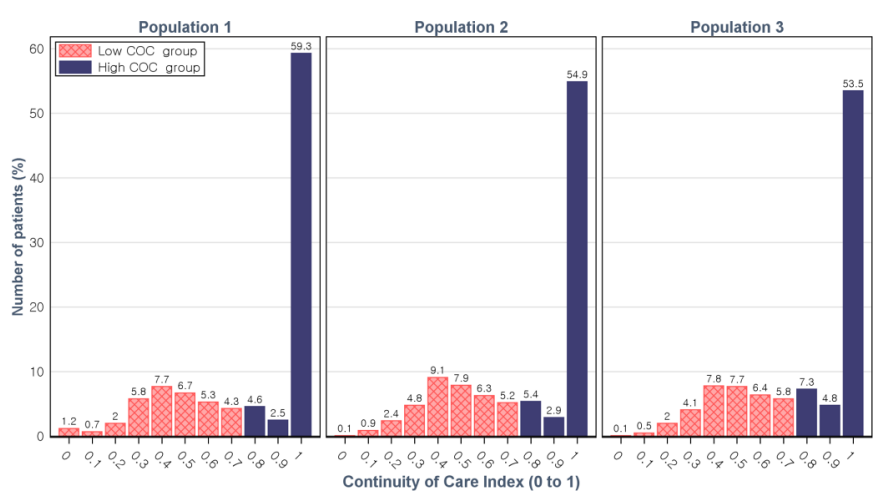

Figure 2 Continuity of Care Index distribution by population during the first 3 years. $\mathrm{COC}$, continuity of care. 
attack, diagnosed with codes G45.0-3 and G45.8-9 and assessed by brain imaging tests (CT or MRI).

\section{Covariates}

Patient characteristics such as sex, age, insurance type (payer), insurance contribution, urbanisation level of the patient's residence and comorbidity were considered as covariates. Patient age, insurance type and level of contribution, and living area were captured as those of the index date. In Korea, there are two types of insurance: NHI and Medical Aid. ${ }^{15}$ Insurance contribution was classified into three categories (high, moderate and low). Urbanisation level was divided into three categories (large urban, small urban and rural). Classifications of insurance contribution and urbanisation level reflected the common notion in the Korean society. As a proxy of the study subject's health status at the time of indexing, the Elixhauser Comorbidity Index (ECI) and the presence of hypertension (I10-I15) or diabetes (E10-E14) were determined based on patients' outpatient and inpatient records during the history period. The ECI for each patient was calculated using Quan et $a l^{\text {s }}$ work $^{16}$ and was grouped into four groups $(0,1,2$ and $\geq 3)$ considering the quartile from the ECI score distribution.

\section{Statistical analysis}

Patient characteristics, COCI, number of visits and number of ASCVD-related hospitalisation according to the COC group were summarised as mean and SD, median and IQR for continuous variables, and as frequency and percentage for categorical variables. Patient characteristics were compared according to the level of continuity of care using the Wilcoxon rank-sum test for continuous variables because normality assumption was not satisfied and $\chi^{2}$ test for the categorical variables. The association between continuity and health outcome was investigated using the Cox proportional hazard regression model. The COCI was considered as a time-dependent exposure in T3, but not in T1, T2 and T4. Crude Harzard Ratios (HRs) and adjusted HRs (after adjusting for age, sex, comorbidity as Elixhauser score, urbanisation level and insurance contribution) were presented with $95 \%$ Confidence Intervals (CIs). The proportional assumption of the Cox proportional hazards model was checked by examining the cumulative Martingale residuals plots and the Kolmogorov-type supremum test. Kaplan-Meier survival plots of ASCVD-related hospitalisation were constructed, and the $\mathrm{p}$ values from the log-rank test for $\mathrm{T} 1, \mathrm{~T} 2$ and $\mathrm{T} 4$ and the crude HR for T3 were presented. All statistical analyses were performed using the SAS statistical software package (V.9.4) and $\mathrm{p}<0.05$ was considered significant.

\section{RESULTS}

\section{Study population}

Figure 3 presents the process and the results of study population selection. Patients were specified according to the selection criteria and a small number of patients whose age was unknown were excluded. Finally, 4754-10 084 patients were included in each population group for analyses.

Table 1 summarises the patient characteristics of the three population groups, P1 ( $\mathrm{n}=10084), \mathrm{P} 2(\mathrm{n}=8454)$ and P3 $(n=4754)$. There were more women than men across all three populations. P3 had the highest share of women and seniors ( 65 older), followed in order by P2 and P1. Subjects in P3 were more likely to have hypertension or diabetes as a comorbidity and showed higher ECI scores than those in P2 and P1. On average, each patient had about 0.8 COCI during the first 3 years, which decreased to 0.75 in $\mathrm{P} 3$ when calculated for the seven study years. The decline was more in P1 and P2, decreasing from 0.8 to about 0.72 .

As the definition of temporal relationship between continuity and outcome dictated the period of continuity measurement, the composition of the COC cohorts in each population group was different. Patient characteristics according to COC level in each study scenario are available in online supplemental tables S1 (T1), S2 (T2) and S3 (T4). In scenario T3, patients in the two COC groups changed with time; thus, the number of patients in the two COC groups could not be determined. There were more women than men in the low COC group across $\mathrm{T} 1, \mathrm{~T} 2$ and T4 in all three populations. Subjects in the high COC group were more likely to have hypertension or diabetes as a comorbidity and showed higher ECI scores across T1, T2 and T4 in all populations. In scenarios P1T4 and P3T4, although there were significant differences in

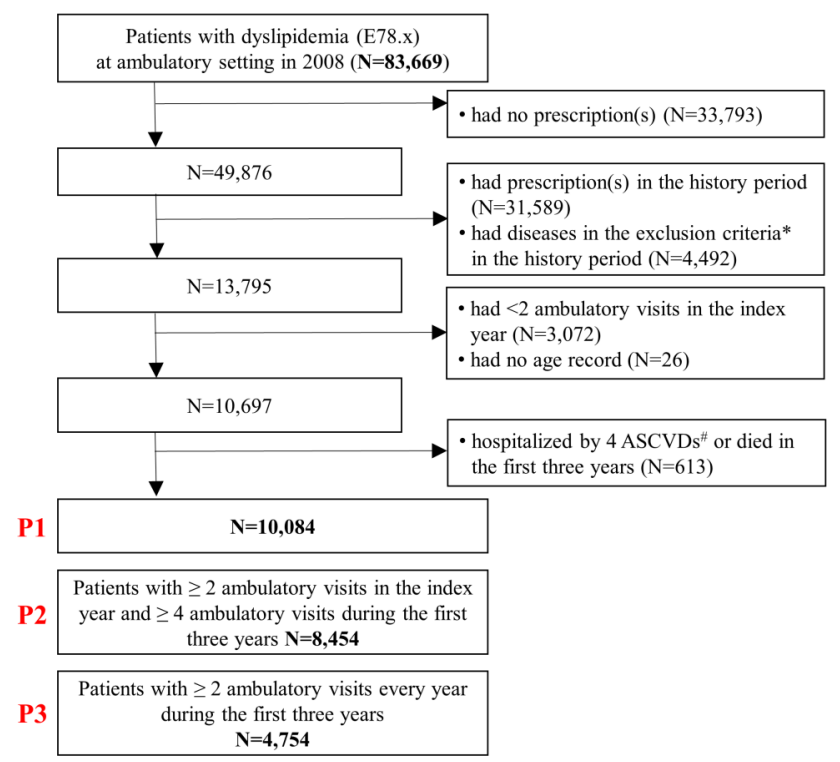

Figure 3 Selection of the study population. *Ischaemic heart diseases (I20-I25), cerebrovascular diseases and related syndromes (160-169, G45-G46), diabetes mellitus with circulatory complications (E10.5, E11.5, E12.5, E13.5, E14.5) and cancer (C00-C97). \#Myocardial infarction (I21.0-4, I21.9, I22.0-1, I22.8-9), stable or unstable angina (I20), ischaemic stroke (163.0-6, I63.8-9) and transient ischaemic attack (G45.0-3, G45.8-9). ASCVD, atherosclerotic cardiovascular disease. 
Table 1 Patient characteristics by study population

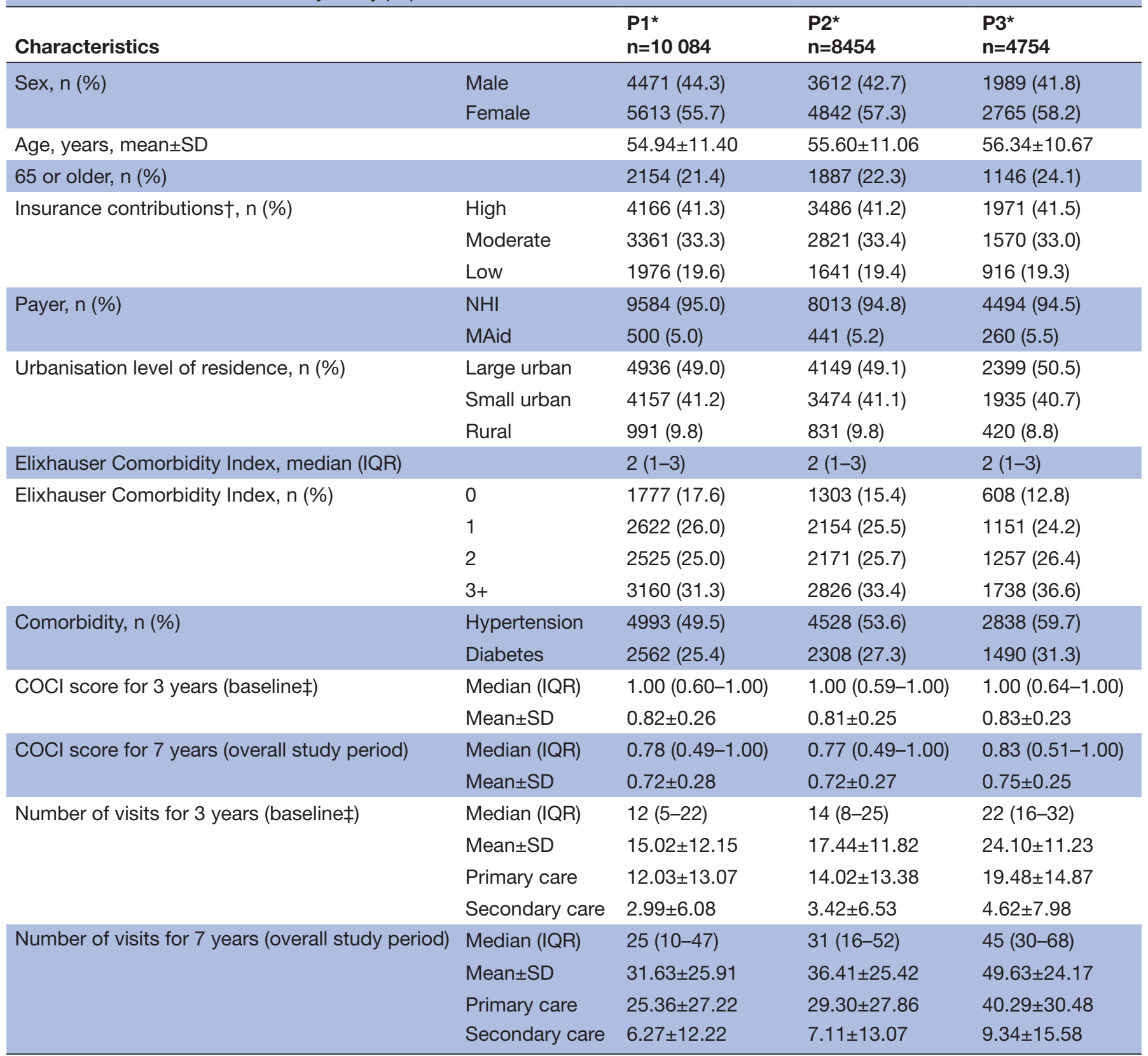

*P1: patients who made at least two visits with a doctor during the first 1 year; P2: patients who made at least two visits with a doctor during the first 1 year and at least four visits during the first 3 years; P3: patients who made at least two visits with a doctor every year during the first 3 years.

†Information for insurance contribution was missing for 581 (5.8\%) patients in P1, 506 (6.0\%) in P2 and 297 (6.3\%) in P3. $\ddagger$ Baseline refers to the first 3 years.

$\mathrm{COCl}$, Continuity of Care Index; MAid, Medical Aid; NHI, National Health Insurance.

age between the groups, it was not considered to be of practical significance. There were no significant differences between the two COC groups across all analysis scenarios by other covariates, including insurance contribution, payer and urbanisation level of the resident area.

Risk of ASCVD-related hospitalisation by level of continuity of care according to analysis scenarios

The probability of event-free by population and temporal relationship scenarios is plotted in figure 4 . In the $\mathrm{T} 1$,
T3 and P3T4 scenarios, the probability of event-free was significantly different between the two COC groups, while in the other scenarios there were no differences between the two groups.

Table 2 presents the number of patients having ASCVDrelated hospitalisation according to COC level in each scenario. In scenario T3, patients in the two COC groups changed with time; thus, the number of patients with the outcome could not be determined. In the T1 scenario, 


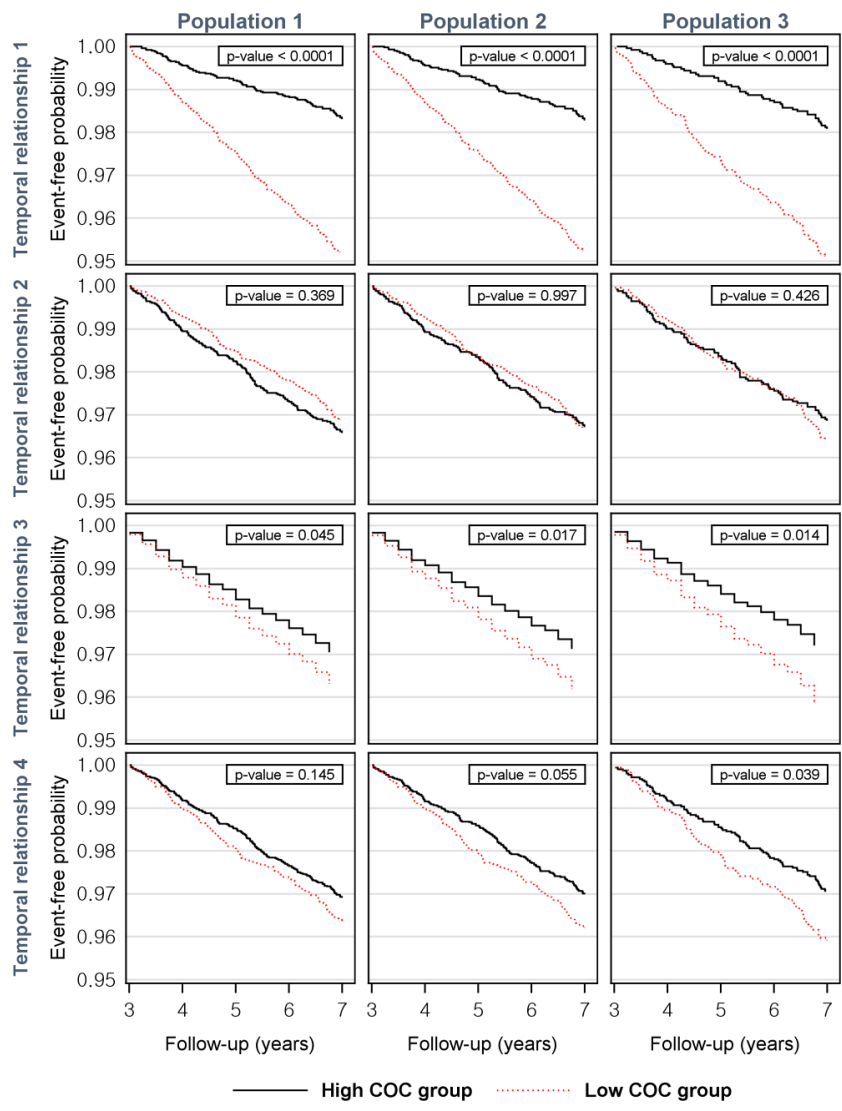

Figure 4 Probability of event-free by population and temporal relationship scenarios. Event: ASCVD-related hospitalisation. ASCVD, atherosclerotic cardiovascular disease; COC, continuity of care.

patients in the low COC group were more likely to have an ASCVD-related hospitalisation than those in the high COC group in all three populations, with the adjusted HR ranging from 2.71 to 3.05. The temporal relationship T2 showed no risk differences for ASCVD-related hospitalisation between the two COC groups in all populations. For $\mathrm{T} 3$ in all populations and $\mathrm{T} 4$ in $\mathrm{P} 2$ and $\mathrm{P} 3$, the low COC groups were more likely to have ASCVD-related hospitalisation. T3 had an adjusted HR of 1.31 (95\% CI 1.05 to 1.63 ) in $\mathrm{P} 1,1.43$ (95\% CI 1.12 to 1.81 ) in $\mathrm{P} 2$ and 1.55 (95\% CI 1.13 to 2.13) in P3. T4 had an adjusted HR of 1.34 (95\% CI 1.05 to 1.70$)$ in P2 and 1.46 (95\% CI 1.06 to 2.01) in P3.

Sensitivity analysis was applied to the three populations with the temporal relationship T4 using the median COCI as a cohort cut-point (table 3). In all populations, the risk of ASCVD-related hospitalisation for the low COC group was more significant than those obtained when the cohorts were divided at $\mathrm{COCI}=0.8$.

\section{DISCUSSION}

This study explored how the definition of the elements within a COC study's design can affect the results in COC studies that assess the association between continuity and outcomes in patients with dyslipidaemia. While there have been several studies discussing the possible effects of temporal relationships on the continuity and outcome measurements, this is the first study to employ empirical data to demonstrate the magnitude of the potential bias that could arise due to an inadequate definition of the relative timing between continuity and outcome measures.

Across the three populations in the study, the concurrent measure of continuity and outcomes (T1) tended to inflate the risk of ASCVD-related hospitalisation by twofold to threefold compared with the risks in the three other temporal relationship strategies. In South Korea, an ASCVD event could decrease continuity, rather a decrease in continuity increasing the chance of an event, as institutional transfers usually occur when a patient has been hospitalised due to one of four ASCVDs. A patient who experiences a poor outcome may be more likely to be classified into the low COC group; as a result, the risk of an event could be exaggerated incorrectly in the low COC group. In such a case, measurements of the continuity after an outcome event will lead to a reverse conclusion, as van Walraven and colleagues ${ }^{5}$ warned in their review. This is a typical bias caused by the cross-sectional study design that measures the cause and effect simultaneously and fails to determine which of the two causes the other.

Continuity being measured until a quarter prior to the outcome occurring (T2) concluded that there was an insignificant association between continuity and outcomes. In this temporal relationship between continuity and outcome, the continuity measuring duration varied widely among patients. Moreover, the later a patient experienced an event, the longer the continuity was measured. For example, a patient with no event was measured for continuity during the entire study period. Previous research has demonstrated that the level of continuity appears to decrease with time as the number of visits increases ${ }^{17}$ a pattern that was repeatedly observed in this analysis. This means that patients with no events are more likely to fall into the low COC group, thereby reducing the risk difference between the two COC groups.

As discussed above, the first and second temporal relationship strategies have temporal ambiguity that can be a source of bias. The other two strategies, in which continuity and outcome were measured concurrently with continuity as a time-varying variable (T3), or continuity was determined over a period followed by a period during which outcomes were measured (T4), have a similar pattern of risk of hospitalisation. Based on the T3 and T4 results, we made a conclusion that the provider COC might be positively related to reducing the risk of ASCVDrelated hospitalisation in patients with dyslipidaemia.

The differences across study populations (P1-P3) were interesting. As the inclusion criteria became stricter, the effect estimates became more substantial. The risk of ASCVD-related hospitalisation was higher in P3 than in P2 or P1, suggesting that the exclusion level for low users can affect the results. This is likely to be related to nondifferential misclassification, which typically dilutes the 
Table 2 Risk of ASCVD-related hospitalisation by level of continuity of care

\section{Patients with ASCVD-}

related hospitalisation,

\begin{tabular}{|c|c|c|c|c|c|c|c|}
\hline \multirow[b]{2}{*}{$\begin{array}{l}\text { Study } \\
\text { population }\end{array}$} & \multirow[b]{2}{*}{$\begin{array}{l}\text { Temporal } \\
\text { relationship }\end{array}$} & \multicolumn{2}{|c|}{ Overall patients, $\mathrm{n}(\%)$} & \multicolumn{2}{|c|}{ n (\%) } & \multicolumn{2}{|c|}{ HR (95\% CI) (reference: high COC group) } \\
\hline & & $\begin{array}{l}\text { Low COC } \\
\text { group }\end{array}$ & $\begin{array}{l}\text { High COC } \\
\text { group }\end{array}$ & $\begin{array}{l}\text { Low COC } \\
\text { group }\end{array}$ & $\begin{array}{l}\text { High COC } \\
\text { group }\end{array}$ & Crude HR & Adjusted HR \\
\hline \multirow{4}{*}{$\begin{array}{l}\mathrm{P} 1 \dagger \\
(\mathrm{n}=10084)\end{array}$} & T1 & $5114(50.7)$ & $4970(49.3)$ & $243(4.8)$ & $83(1.7)$ & $2.88(2.25 \text { to } 3.70)^{\star \star \star}$ & 3.05 (2.38 to 3.92$)^{\star \star \star}$ \\
\hline & T2 & $5026(49.8)$ & $5058(50.2)$ & $155(3.1)$ & $171(3.4)$ & 0.91 (0.73 to 1.13$)$ & 0.96 (0.77 to 1.19$)$ \\
\hline & T3 & NA & NA & NA & NA & $1.25(1.01 \text { to } 1.56)^{*}$ & $1.31(1.05 \text { to } 1.63)^{*}$ \\
\hline & $\mathrm{T} 4$ & $3397(33.7)$ & $6687(66.3)$ & $122(3.6)$ & $204(3.1)$ & 1.18 (0.94 to 1.48$)$ & 1.22 (0.97 to 1.52$)$ \\
\hline \multirow{4}{*}{$\begin{array}{l}\mathrm{P} 2 \dagger \\
(\mathrm{n}=8454)\end{array}$} & T1 & $4389(51.9)$ & $4065(48.1)$ & $206(4.7)$ & $69(1.7)$ & $2.80(2.13 \text { to } 3.68)^{\star \star \star}$ & $3.01(2.29 \text { to } 3.95)^{\star * \star}$ \\
\hline & T2 & $4324(51.1)$ & $4130(48.9)$ & $141(3.3)$ & $134(3.2)$ & $1.00(0.79$ to 1.27$)$ & 1.07 (0.85 to 1.36$)$ \\
\hline & T3 & NA & NA & NA & NA & $1.34(1.05 \text { to } 1.69)^{*}$ & $1.43(1.12 \text { to } 1.81)^{\star \star}$ \\
\hline & $\mathrm{T} 4$ & $3104(36.7)$ & $5350(63.3)$ & $116(3.7)$ & $159(3.0)$ & 1.26 (0.995 to 1.61$)$ & $1.34(1.05 \text { to } 1.70)^{\star}$ \\
\hline \multirow{4}{*}{$\begin{array}{l}\text { P3† } \\
(n=4754)\end{array}$} & T1 & $2271(47.8)$ & $2483(52.2)$ & $110(4.8)$ & $47(1.9)$ & $2.60(1.85 \text { to } 3.66)^{\star * \star}$ & $2.71(1.93 \text { to } 3.82)^{\star \star \star}$ \\
\hline & T2 & $2240(47.1)$ & $2514(52.9)$ & $75(3.5)$ & $78(3.1)$ & 1.14 (0.83 to 1.55$)$ & 1.18 (0.86 to 1.62$)$ \\
\hline & T3 & NA & NA & NA & NA & $1.49(1.09 \text { to } 2.03)^{*}$ & $1.55(1.13 \text { to } 2.13)^{\star \star}$ \\
\hline & T4 & 1635 (34.4) & $3119(65.6)$ & $66(4.0)$ & $91(2.9)$ & $1.40(1.02 \text { to } 1.92)^{\star}$ & $1.46(1.06 \text { to } 2.01)^{\star}$ \\
\hline
\end{tabular}

${ }^{*} \mathrm{P}<0.05,{ }^{* *} \mathrm{P}<0.01,{ }^{\star * *} \mathrm{P}<0.001$.

†P1: patients who made at least two visits with a doctor during the first 1 year; P2: patients who made at least two visits with a doctor during the first 1 year and at least four visits during the first 3 years; P3: patients who made at least two visits with a doctor every year during the first 3 years.

†The adjusted HR was analysed after adjusting for covariates including sex, age, insurance contribution, payer, urbanisation level of resident area and comorbidity as Elixhauser score.

ASCVD, atherosclerotic cardiovascular disease; COC, continuity of care; NA, not available.

associations between exposures and outcomes. Patients with few visits may be difficult to classify properly. Setting the inclusion criterion to include more visits allows the researcher to set up an experimental-likely environment that could exclude patients with ambiguous care continuity due to a small number of visits. When the P3 criterion was applied, $43 \%$ of the study subjects in P2 were eliminated. Of these, $83 \%$ had no visits in the second or third year. In South Korea, it has been reported that compliance of patients with dyslipidaemia was likely to be moderate. Sometimes, they revisited a doctor's office some years later after being diagnosed. ${ }^{18}$ Hence, the P2 population of this study was considered to represent an actual population, increasing the generalisability to real-world

Table 3 Risk of ASCVD-related hospitalisation by cohort cut-point

\begin{tabular}{|c|c|c|c|c|c|c|c|}
\hline \multirow[b]{2}{*}{ Scenario } & \multirow{2}{*}{$\begin{array}{l}\mathrm{COCl} \text { at } \\
\text { cohort cut- } \\
\text { point }\end{array}$} & \multicolumn{2}{|c|}{ Overall patients, n (\%) } & \multicolumn{2}{|c|}{$\begin{array}{l}\text { Patients with ASCVD-related } \\
\text { hospitalisation, } n(\%)\end{array}$} & \multicolumn{2}{|c|}{$\begin{array}{l}\text { HR (95\% Cl) (reference: high COC } \\
\text { group) }\end{array}$} \\
\hline & & $\begin{array}{l}\text { Low COC } \\
\text { group }\end{array}$ & $\begin{array}{l}\text { High COC } \\
\text { group }\end{array}$ & $\begin{array}{l}\text { Low COC } \\
\text { group }\end{array}$ & $\begin{array}{l}\text { High COC } \\
\text { group }\end{array}$ & Crude HR & Adjusted HR \\
\hline \multirow{2}{*}{$\begin{array}{l}\mathrm{P} 1 \mathrm{~T} 4 \dagger \\
(\mathrm{n}=10084)\end{array}$} & 0.8 & 3397 (33.7) & 6687 (66.3) & $122(3.6)$ & $204(3.1)$ & $1.18(0.94$ to 1.48$)$ & 1.21 (0.97 to 1.52$)$ \\
\hline & Median (1.0) & $4105(40.7)$ & 5979 (59.3) & $151(3.7)$ & $175(2.9)$ & $1.26(1.02 \text { to } 1.57)^{*}$ & $1.26(1.01 \text { to } 1.57)^{\star}$ \\
\hline \multirow{2}{*}{$\begin{array}{l}\mathrm{P} 2 \mathrm{~T} 4 \dagger \\
(\mathrm{n}=8454)\end{array}$} & 0.8 & $3104(36.7)$ & 5350 (63.3) & $116(3.7)$ & $159(3.0)$ & 1.26 (0.995 to 1.61$)$ & $1.34(1.05 \text { to } 1.70)^{*}$ \\
\hline & Median (1.0) & $3812(45.1)$ & 4642 (54.9) & $145(3.8)$ & $130(2.8)$ & $1.37(1.08 \text { to } 1.73)^{*}$ & $1.41(1.11 \text { to } 1.79)^{\star \star}$ \\
\hline \multirow{2}{*}{$\begin{array}{l}\text { P3T4† } \\
(n=4754)\end{array}$} & 0.8 & $1635(34.4)$ & 3119 (65.6) & $66(4.0)$ & $91(2.9)$ & $1.40(1.02 \text { to } 1.92)^{*}$ & $1.46(1.06 \text { to } 2.01)^{\star}$ \\
\hline & Median (1.0) & 2209 (46.5) & 2545 (53.5) & $88(4.0)$ & $69(2.7)$ & $1.48(1.08 \text { to } 2.03)^{\star}$ & $1.51(1.10 \text { to } 2.07)^{\star}$ \\
\hline
\end{tabular}

${ }^{\star} \mathrm{P}<0.05,{ }^{* \star} \mathrm{P}<0.01,{ }^{* \star *} \mathrm{P}<0.001$.

†P1: patients who made at least two visits with a doctor during the first 1 year; P2: patients who made at least two visits with a doctor during the first 1 year and at least four visits during the first 3 years; P3: patients who made at least two visits with a doctor every year during the first 3 years; T4: measuring continuity for the first 3 years and measuring outcomes in the remaining period. $\ddagger$ The adjusted HR was analysed after adjusting for covariates including sex, age, insurance contribution, payer, urbanisation level of resident area and comorbidity as Elixhauser score.

ASCVD, atherosclerotic cardiovascular disease; COC, continuity of care; $\mathrm{COCl}$, continuity of care index. 
patients. In P1, nearly $5 \%$ of the patients made only two visits during the study period. One potential explanation for the low number of visits was that they may have received a false diagnosis in the index year, meaning they might not have been patients with dyslipidaemia. Among two-visit subjects, $89 \%$ had a COCI value of 1 and were classified into the high COC group. If some of them were not patients with dyslipidaemia, they likely decreased the risk of event in the high COC group, leading to a faulty conclusion.

The present study employing real-world data had several limitations due to restrictions of claims data. The claims data are collected for administrative purposes and provide no information on non-administrative features such as trust, patient experience or satisfaction, factors that may determine the quality of a relationship. Hence even though provider-patient continuity encompasses several aspects of human relationships, the present study investigated only limited facets of those relationships due to data unavailability. In addition, there was no information on the informational links between different providers who offered care consults to the same patient.

Another important limitation was that the anonymised claims data do not have information on individual health providers. To overcome this limitation, this study considered a visit to the same hospital and a visit to the same medical department within the hospital environment when defining a visit to the same provider. This is because, first, most patients of such facilities will, whenever possible, visit the doctors they have previously seen and, second, informational continuity is expected within a single hospital. However, this might overestimate the COCI score by misclassifying patients with low continuity into the high continuity group, and as a result reducing the difference of the risk of ASCVD-related hospitalisation between the two groups. However, the shares of secondary care in three study populations were about 20\%; hence, the effects of such misclassification are expected to be limited. Furthermore, as mentioned earlier, the present study analysed claims data to measure continuity of care using the COCI, reflecting only one aspect, that is, dispersion of the provider-patient relationship. It should be noted that other measures of COC can reflect the different aspects of the physician-patient relationships: duration, density, dispersion and sequence. ${ }^{14}$ For instance, in short-term assessments, a sequence type index (eg, SECON) may be a good choice to investigate the association of continuity with chronic conditions. ${ }^{13} \mathrm{In}$ other instances where patient-level data are available, it may be possible to identify the usual provider for each patient. In such situations, a density-type index (eg, the UPC index) could enable richer interpretations of the study findings. ${ }^{14}$

There are a few things to consider when generalising our findings. The Korean healthcare system provides universal coverage and patients are free to choose care providers with few legal restrictions, which could substantially affect how people use the healthcare service. Our assumption that most patients attending hospitals will visit the doctor they have previously seen may not be the same as other countries with different medical cultures. The informational continuity in Korean facilities, which have robust electronic systems, may not be assumed in other healthcare systems that do not have electronic records. The nature of the disease is another factor that potentially affects the use of healthcare services. Thus, caution should be exercised when applying these findings to data generated by other healthcare systems or other diseases.

\section{CONCLUSIONS}

The results of continuity of care studies are sensitive to the population selection criteria and the temporal relationship between continuity and outcome. Thus, researchers should be cautious of these when planning continuity studies. The concurrent measure of continuity and outcome could exaggerate the risk of an outcome event by measuring the cause and effect simultaneously. In the selection of study population, including extreme low users could dilute the associations between continuity and outcome if there is failure in the classification of patients.

Acknowledgements This study was conducted using data from the Korean National Health Insurance Service (research management number NHIS-2020-2-129).

Contributors I-HL: a guarantor of the study, conceptualisation of the study, statistics, drafting of the manuscript, funding acquisition. EJJ: conceptualisation of the study, statistical consultations, critical revision of the manuscript. EChoo: conceptualisation of the study, statistics, drafting of the manuscript. EChoi: acquisition of NHIS-NSC data, conceptualisation of the study, critical revision of the manuscript. JL: statistics. LS: drafting of the manuscript. All authors read and approved final version of the manuscript.

Funding This work was supported by a National Research Foundation of Korea (NRF) grant funded by the Korea government (MSIT) (grant number 2020R1A2C1005061.

Competing interests None declared.

Patient consent for publication Not required.

Ethics approval This study was reviewed by the Institutional Review Board of Yeungnam University (YU201804004002). The requirement for informed consent was waived because this study analysed anonymous claims data provided for research purposes by the Korean National Health Insurance Service.

Provenance and peer review Not commissioned; externally peer reviewed.

Data availability statement Data may be obtained from a third party and are not publicly available. Raw data that support the findings of this study are available only for authorised researchers in South Korea and only for a limited period due to the information protection law for patient privacy.

Supplemental material This content has been supplied by the author(s). It has not been vetted by BMJ Publishing Group Limited (BMJ) and may not have been peer-reviewed. Any opinions or recommendations discussed are solely those of the author(s) and are not endorsed by BMJ. BMJ disclaims all liability and responsibility arising from any reliance placed on the content. Where the content includes any translated material, BMJ does not warrant the accuracy and reliability of the translations (including but not limited to local regulations, clinical guidelines, terminology, drug names and drug dosages), and is not responsible for any error and/or omissions arising from translation and adaptation or otherwise.

Open access This is an open access article distributed in accordance with the Creative Commons Attribution Non Commercial (CC BY-NC 4.0) license, which permits others to distribute, remix, adapt, build upon this work non-commercially, and license their derivative works on different terms, provided the original work is 
properly cited, appropriate credit is given, any changes made indicated, and the use is non-commercial. See: http://creativecommons.org/licenses/by-nc/4.0/.

\section{ORCID iD}

lyn-Hyang Lee http://orcid.org/0000-0001-6644-0808

\section{REFERENCES}

1 Pereira Gray DJ, Sidaway-Lee K, White E, et al. Continuity of care with Doctors-a matter of life and death? A systematic review of continuity of care and mortality. BMJ Open 2018;8:e021161.

2 Kao Y-H, Lin W-T, Chen W-H, et al. Continuity of outpatient care and avoidable hospitalization: a systematic review. Am J Manag Care 2019;25:e126-34.

3 Saultz JW. Defining and measuring interpersonal continuity of care. Ann Fam Med 2003;1:134-43.

4 Bentler SE, Morgan RO, Virnig BA, et al. Do claims-based continuity of care measures reflect the patient perspective? Med Care Res Rev 2014;71:156-73.

5 van Walraven $\mathrm{C}$, Oake N, Jennings $\mathrm{A}$, et al. The association between continuity of care and outcomes: a systematic and critical review. $J$ Eval Clin Pract 2010;16:947-56.

6 Robles S, Anderson GF. Continuity of care and its effect on prescription drug use among Medicare beneficiaries with hypertension. Med Care 2011:49:516-21.

7 Dreiher J, Comaneshter DS, Rosenbluth Y, et al. The association between continuity of care in the community and health outcomes: a population-based study. Isr J Health Policy Res 2012;1:21.

8 Romano MJ, Segal JB, Pollack CE. The association between continuity of care and the overuse of medical procedures. JAMA Intern Med 2015;175:1148-54.
9 Cho KH, Park E-C, Nam YS, et al. Impact of market competition on continuity of care and hospital admissions for asthmatic children: a longitudinal analysis of nationwide health insurance data 2009-2013. PLoS One 2016;11:e0150926.

10 Barker I, Steventon A, Deeny SR. Association between continuity of care in general practice and hospital admissions for ambulatory care sensitive conditions: cross sectional study of routinely collected, person level data. BMJ 2017;356:j84.

11 Bice TW, Boxerman SB. A quantitative measure of continuity of care. Med Care 1977:15:347-9.

$12 \mathrm{NHIS} \mathrm{H.} \mathrm{National} \mathrm{health} \mathrm{insurance} \mathrm{statistical} \mathrm{yearbook} \mathrm{2019.} \mathrm{Seoul:}$ National Health Insurance Service, Health Insurance Review \& Assessment, 2020

13 Steinwachs DM. Measuring provider continuity in ambulatory care: an assessment of alternative approaches. Med Care 1979;17:551-65.

14 Jee SH, Cabana MD. Indices for continuity of care: a systematic review of the literature. Med Care Res Rev 2006;63:158-88.

15 National Health Insurance Service. National health insurance Wonju, 2021. Available: https://www.nhis.or.kr/english/index.do\# [Accessed 8 Apr 2021].

16 Quan H, Sundararajan V, Halfon P, et al. Coding algorithms for defining comorbidities in ICD-9-CM and ICD-10 administrative data. Med Care 2005;43:1130-9.

17 Hong JS, Kang HC, Kim J. Continuity of care for elderly patients with diabetes mellitus, hypertension, asthma, and chronic obstructive pulmonary disease in Korea. J Korean Med Sci 2010;25:1259-71.

18 KSoLA. Dyslipidemia fact sheets in Korea 2018. Seoul: The Korean Society of Lipid and Atherosclerosis, 2018. 